\title{
A COMPARATIVE STUDY OF LAPAROSCOPIC TEP AND OPEN LICHTENSTEIN'S TENSION-FREE HERNIA REPAIR: A SINGLE SURGICAL UNIT EXPERIENCE
}

\author{
Ershad Hussain Galeti1, Ramanji Gundluru2, Gousia Begum Sowdagar ${ }^{3}$ \\ ${ }^{1}$ Assistant Professor, Department of General Surgery, PES Medical College, Kuppam. \\ ${ }^{2}$ Postgraduate, Department of General Surgery, PES Medical College, Kuppam. \\ 3Postgraduate, Department of Anaesthesia, PES Medical College, Kuppam.
}

\begin{abstract}
\section{BACKGROUND}

This is a prospective study involving 50 cases of unilateral inguinal hernia, 25 of whom underwent open Lichtenstein's repair and the remaining, Laparoscopic Total Extraperitoneal (TEP) Repair. The study deals with a comparison of the effectiveness of these two types of repair.
\end{abstract}

\section{MATERIALS AND METHODS}

50 patients of unilateral inguinal hernia admitted in PES Medical College, Kuppam, Andhra Pradesh, India, were selected on the basis of convenient sampling method. Adults (>18 years) consenting for randomised trials and having either unilateral direct or indirect inguinal hernia were included. They were randomly chosen for Lichtenstein's or TEP repair and the results were compared on the basis of the following outcome measures: 1) Duration of surgery (In min.), 2) Postoperative pain (In days), 3) Length of hospital stay (In days), 4) Post-operative complications like seroma, scrotal oedema, urinary retention, wound infection, 5) Cost comparison, 6) Recurrence rate.

\section{RESULTS}

The outcomes were evaluated and compared with standard published literature. The mean duration of surgery for Lichtenstein's repair was 58.5 minutes whereas for TEP was 74.6 minutes. Duration of post-operative pain was more (2.9 days) for open repair than 1.3 days for TEP repair. Post-operative stay was longer (5.1 days) for open repair than 1.6 days for TEP. Minimal complications were noted in Laparoscopic TEP group (One incidence of seroma) as compared to the open Lichtenstein's hernioplasty (Postoperative seroma, scrotal oedema, urinary retention \& wound infection). Recurrence was not seen with the open Lichtenstein's repair, which was seen in 1 patient of the laparoscopic TEP, which was managed by the Lichtenstein repair. However, TEP repair was approximately Rs. 4000 more expensive than open Lichtenstein repair. TEP repair, though expensive, is superior with regard to reduced post-operative pain, reduced hospital stay and minimal complications, when compared to Lichtenstein's repair.

\section{CONCLUSION}

The present study is a comparison of the effectiveness and complications of the Lichtenstein's tension-free repair and the Total Extraperitoneal (TEP) Repair. All the patients were carefully monitored from the time of admission till discharge, and the parameters pertaining to the study noted. We found that there is a marked reduction in post-operative pain in laparoscopic hernia repair compared to hernioplasty. Post-operative stay is less in TEP repair. Laparoscopic hernia repair is quite expensive compared to hernioplasty. Despite the fact that TEP is a demanding procedure, it may be performed efficiently with an acceptable operating time and a low complication rate. Although there is a very long learning curve for Laparoscopic TEP, but still it is a preferred technique for the management of inguinal hernia repair in an experienced hand.

\section{KEYWORDS}

TEP, Lichtenstein's Repair, Inguinal Hernia.

HOW TO CITE THIS ARTICLE: Galeti EH, Gundluru R, Sowdagar GB. A comparative study of laparoscopic TEP and open Lichtenstein's tension-free hernia repair: A single surgical unit experience. J. Evolution Med. Dent. Sci. 2016;5(80):5956-5958, DOI: $10.14260 /$ jemds/2016/1345

\section{BACKGROUND}

Open repair has been the standard surgical approach for the treatment of inguinal hernia. Use of mesh with the open technique reduces recurrence between 50 and $75 \%$. In the past 20 years, laparoscopic techniques have been introduced for the treatment of hernias that include Trans-abdominal pre

Financial or Other, Competing Interest: None.

Submission 13-09-2016, Peer Review 24-09-2016,

Acceptance 27-09-2016, Published 05-10-2016.

Corresponding Author:

Dr. Ershad Hussain Galeti,

Noble Hospital, LBS Road,

Pileru-517214.

E-mail: dr.ershadhussain@gmail.com

DOI: $10.14260 / \mathrm{jemds} / 2016 / 1345$

\section{(c) (i) $\ominus$}

peritoneal method (TAPP), and Total extra-peritoneal approach (TEP).1,2 As the NICE guidelines for treatment of hernias, 2004, laparoscopic surgery should be offered as one of the treatment options for inguinal hernias. ${ }^{3}$

In our institution, inguinal hernia repair is one of the most common surgeries performed. The procedures performed most commonly are open Lichtenstein's tension-free repair and laparoscopic total extra-peritoneal repair (TEP). This study aims to find which surgery, among these two, is more advantageous.

\section{AIMS AND OBJECTIVES}

To compare laparoscopic extra-peritoneal repair (TEP) with open Lichtenstein's repair on the basis of i) Duration of operation (In mins.), ii) Post-operative pain (In days), iii) 
Length of hospital stay (In days), iv) Post-Operative complications like seroma, v) Cost comparison, vi) Recurrence rate.

\section{MATERIALS AND METHODS}

The present study is a prospective study of fifty cases of unilateral inguinal hernia admitted in PES Medical College and Hospital, Kuppam, India during the period from October 2014 to April 2016. 50 cases were selected on the basis of convenience sampling method. Both direct and indirect unilateral inguinal hernias were selected. After pre-operative preparation, the patients were selected randomly for Lichtenstein's hernia repair or TEP.

\section{Inclusion Criteria}

Adults (18 years) consenting for randomised trials, Unilateral direct or indirect inguinal hernia.

\section{Exclusion Criteria}

Congenital hernias, Recurrent hernias, Bilateral inguinal hernias, patients who have already had lower abdominal surgery, people who are not fit for GA- COPD, Bronchial asthma (ASA GRADE >III).

All patients included in the study were evaluated with a thorough history and physical examination. Routine investigations were done. Ultrasound abdomen and prostate specific antigen tests were done in the ones whom these were indicated.

In laparoscopic TEP group, pre peritoneal space was entered just below the umbilicus and enlarged using gentle blunt dissection with a laparoscope. Two $5 \mathrm{~mm}$ ports were placed in the midline under direct vision, and reusable cannulas and instruments were used. After the hernia sac was reduced, a $15 \times 10 \mathrm{~cm}$ polypropylene mesh was used to cover the myopectineal orifice in all patients and was fixed to the pectineal ligament and further laterally using tacks. Open Lichtenstein repair was done with use of $11 \times 6 \mathrm{~cm}$ polypropylene mesh. A single dose of prophylactic preoperative antibiotic cefazolin was given with one dose post-operatively. Analgesics were given post-operatively.

After surgery, all patients were observed carefully for post-operative pain, duration of hospital stay, any complications were recorded. Post-operative pain was measured on VAS (Visual analogue scale (1-10). The VAS Score was analysed 8 hourly \& the average of them was taken for analysis.

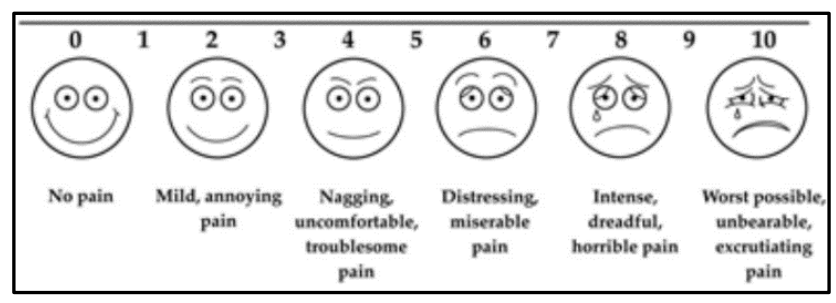

VAS (Visual Analogue Scale)

A careful note was kept as to the duration of the postoperative pain and the patients were discharged when fit. Followup was done first after 2 weeks, and checked for wound infection, persistent pain. Each patient was followed up until 3-6 months till they could return to normal work. The age, surgical treatment, post-operative pain, post-operative complications, cost of the treatment, hospital stay were all evaluated and compared with standard published literature.

\section{RESULTS}

In our study, the youngest patient was 24 years old and the oldest was 72 years old. All patients were men. The youngest patient who underwent Lichtenstein's repair was 28 years old and the oldest was 72 years old and the mean age was 54.76 . The youngest patient who underwent TEP repair was 24 years old and the oldest was 66 years old and the mean age was 52.62. Average duration of Lichtenstein's repair was $58.5 \mathrm{~min}$., 74.6 min. for TEP. The $P$ value is 0.005 , hence significant. Hence, there is an increase in the duration of repair of unilateral TEP repair compared to the Lichtenstein's repair. This may be attributed to the learning curve. Average duration of pain in Lichtenstein's repair was 2.9 days, 1.3 days in TEP repair. $\mathrm{P}$ value is $<0.001$, hence significant. Thus, there is a definite reduction in the duration of post-operative pain (in days) following a TEP than a Lichtenstein's repair. Average duration of post-operative hospital stay in Lichtenstein's repair was 5.1 days, 1.6 days in TEP repair. $P$ value is $<0.001$, hence significant. Thus, patients who underwent Lichtenstein's repair stayed for a longer time in the hospital compared to those who underwent TEP repair. There was only single complication noted in TEP repair patients and it was a seroma which was aspirated ultrasound guided. In Lichtenstein's repair, there were 2 cases of seroma, 2 cases of scrotal oedema, 4 cases of urinary retention and 1 case of wound infection, which were all managed conservatively. Hence, post-operative complication rates are higher in Lichtenstein's repair compared to TEP repair. There is an increased expenditure of Rs. 4000 associated with TEP repair compared to Lichtenstein's repair.

\begin{tabular}{|c|c|c|c|}
\hline Variables & TEP & $\begin{array}{c}\text { Lichtenstein's } \\
\text { Repair }\end{array}$ & $\begin{array}{c}\text { P- } \\
\text { Value }\end{array}$ \\
\hline Mean Age & 52.62 & 54.76 & $>0.05$ \\
\hline $\begin{array}{c}\text { Duration of } \\
\text { Surgery (In min.) }\end{array}$ & 74.6 & 58.5 & 0.005 \\
\hline $\begin{array}{c}\text { Duration of Post- } \\
\text { Operative } \\
\text { Pain (In Days) }\end{array}$ & 1.3 & 2.9 & $<0.001$ \\
\hline $\begin{array}{c}\text { Duration of Post- } \\
\text { Operative Hospital } \\
\text { Stay (In Days) }\end{array}$ & 1.6 & 5.1 & $<0.001$ \\
\hline $\begin{array}{c}\text { Mean Cost } \\
\text { (In Rupees) }\end{array}$ & 8000 & 4000 & $<0.001$ \\
\hline \multicolumn{2}{|c|}{ Comparison of TEP and Lichtenstein's Repair } \\
\hline \multicolumn{4}{|l}{}
\end{tabular}

\begin{tabular}{|c|c|c|}
\hline Complications & TEP & Lichtenstein's Repair \\
\hline Seroma & 1 & 2 \\
\hline Scrotal Oedema & None & 2 \\
\hline Urinary Retention & None & 4 \\
\hline Wound Infection & None & 1 \\
\hline
\end{tabular}

\section{DISCUSSION}

From Bassini's heralding of the modern era to today's meshbased open and laparoscopic repairs, this history parallels closely the evolution in anatomical understanding and development of the techniques of general surgery. 4,5 The fact that more than a hundred repairs have been described for inguinal hernia and practised at some time or the other over 
the past century are a testimony to the fact that none has been considered distinctly superior to the others. In recent years; however, the use of mesh for repair of inguinal hernia has become a norm. Reduction in the recurrence rate from more than $15 \%$ with tissue repairs to less than $1 \%$, reduction in the postoperative pain and a shorter convalescence have all contributed to the popularity and widespread use of the tension-free mesh repairs. The laparoscopic repair of inguinal hernia, a relatively newer modality in the armamentarium of the surgeon, has been around for around two decades. Although perhaps not practised as widely as laparoscopic cholecystectomy is for gallstone disease, laparoscopic repair of inguinal hernia has established its rightful place in the surgical practice. ${ }^{6}$ The advantages of laparoscopic surgery favour its application to various surgical procedures. Better patient comfort, allowing tension-free repair with better exposure to groin anatomy, less postoperative pain, shorter hospital stay and early return to daily activity are some of the advantages of this technique. However, laparoscopic hernioplasty requires lengthy learning curve and it is more costly mainly due to the use of disposables during surgery. Furthermore, with the use of laparoscopic technique, the assessment of both groins, particularly incidental defects, is easier and both defects can be repaired at the same operation without the need of further surgical incision, with very little dissection and minimal additional postoperative discomfort.7,8

Out of the two types of laparoscopic hernia repair followed most commonly, TAPP and TEP, we have exclusively performed TEP repairs in our patients. In TAPP, the surgeon goes into the peritoneal cavity and places a mesh through a peritoneal incision over possible hernia sites. TEP is different as the peritoneal cavity is not entered and mesh is used to seal the hernia from outside the thin membrane covering the organs in the abdomen (The peritoneum). ${ }^{9}$ Initially, TAPP was used and TAPP related some complications were reported previously.10,11,12 The complications noted earlier were minimised and both TAPP and TEP yielded equally better results. But TEP is preferred more than TAPP method presently. There are many comparative studies between TAPP and Lichtenstein tension-free methods but similar studies between TEP and Lichtenstein tension-free methods are limited 13 , and this study presents the results of our experiences between these two techniques that were used.

\section{CONCLUSION}

The present study is a comparison of the effectiveness and complications of the Lichtenstein's tension-free repair and the Total Extraperitoneal (TEP) Repair.

All the patients were carefully monitored from the time of admission till discharge, and the parameters pertaining to the study noted. We found that there is a marked reduction in post-operative pain in laparoscopic hernia repair compared to hernioplasty. Post-operative stay is less in TEP repair. Laparoscopic hernia repair is quite expensive compared to hernioplasty. Despite the fact that TEP is a demanding procedure, it may be performed efficiently with an acceptable operating time and a low complication rate. Although there is a very long learning curve for Laparoscopic TEP, but still it is a preferred technique for the management of inguinal hernia repair in an experienced hand.

\section{REFERENCES}

1. Rosenberg J, Bay-Nielsen M. Current status of laparoscopic inguinal hernia repair in Denmark. Hernia 2008;12(6):583-7.

2. NICE. Implementation uptake report: laparoscopic surgery for inguinal hernia repair London: NICE 2010.

3. NICE. Laparoscopic surgery for inguinal hernia repair. technology appraisal 83. London: NICE 2004.

4. Lau WY. History of treatment of groin hernia. World J Surg 2002;26(6):748-59.

5. Gray SH, Hawn MT, Itani KM. Surgical progress in inguinal and ventral incisional hernia repair. Surg Clin North Am 2008;88(1):17-26.

6. Chowbey PK, Pithawala M, Khullar R, et al. Complications in groin hernia surgery and the way out. J Minim Access Surg 2006;2(3):174-7.

7. Fränneby U, Sandblom G, Nordin P, et al. Risk factors for long-term pain after hernia surgery. Ann Surg 2006;244(2):212-9.

8. Thumbe VJ, Evans DS. To repair or not to repair incidental defects found on laparoscopic repair of groin hernia: early results of a randomized control trail. Surg Endosc 2001;15(1):47-9.

9. Motson RW. Why does NICE not recommend laparoscopic herniorrhaphy? BMJ 2002;324(7345):1092-4.

10. Smith JR, Demers ML, Pollack R, et al. Prospective comparison between laparoscopic preperitoneal herniorrhaphy and open mesh herniorrhaphy. Am Surg 2001;67(2):115-8.

11. Quilici PJ, Greaney EM, Quilici J, et al. Laparoscopic inguinal hernia repair: optimal technical variations and results in 1700 cases. Am Surg 2000;66(9):848-52.

12. Zieren J, Zieren HU, Jacobi CA, et al. Prospective randomized study comparing laparoscopic and open tension-free inguinal hernia repair with Shouldice operation. Am J Surg 1998;175(4):330-3.

13. Johansson B, Hallerback B, Glise H, et al. Laparoscopic mesh versus open preperitoneal mesh versus conventional technique for inguinal hernia repair. Ann Surg 1999;230(2):225-31. 